Génét. Sél. Evol., 1984, 16 (1), 27-44.

\title{
Variabilité génétique du rythme circadien de ponte dans les populations naturelles de Drosophila melanogaster
}

\author{
R. ALLEMAND, P. FOUILLET et J.R. DAVID * \\ Laboratoire de Génétique des Populations (associé au C.N.R.S.) \\ Université Lyon 1, 43, boulevard du 11-Novembre-1918, F 69622 Villeurbanne \\ * Laboratoire de Biologie et Génétique évolutives \\ C.N.R.S., F 91190 Gif-sur-Yvette
}

\begin{abstract}
Résumé
La variabilité génétique du rythme circadien de ponte de $D$. melanogaster a été analysée par la méthode des lignées isofemelles au sein de 2 populations d'origine géographique différentes, l'une afro-tropicale, l'autre française. L'analyse statistique de cette variabilité a conduit à rechercher des indicateurs décrivant et quantifiant les profils de rythme, notamment la phase et l'amplitude des pics; l'un empirique adapté aux courbes de ponte de l'espèce, l'autre plus objectif et plus performant donné par le premier facteur d'une analyse multidimensionnelle (l'analyse des correspondances). Une forte variabilité existe au sein des populations naturelles et entre populations, cependant le rythme de ponte présente pour chacune d'elles des caractéristiques propres, stables au cours du temps. La variabilité intrapopulation donne prise à la sélection artificielle qui conduit à des phénotypes très différents en phase et en amplitude. Une part de la variabilité de l'amplitude du pic maximum pendant l'obscurité provient de celle de la fécondité journalière et s'explique par les capacités physiologiques des femelles à retenir les ovocytes mûrs dans les ovaires en réponse à des déterminants exogènes. Dans les populations naturelles, les caractéristiques du rythme pourraient être maintenues par la sélection naturelle qui agit sur le comportement d'oviposition.
\end{abstract}

Mots clés : Population naturelle, rythme circadien, sélection, analyse multidimensionnelle, D. melanogaster, lignées isofemelles.

\section{Summary}

Genetic variability of circadian rhythm of oviposition in natural populations of Drosophila melanogaster

Using isofemale lines, the genetic variability in the circadian rhythm of oviposition has been analysed within two natural populations of $D$. melanogaster from tropical Africa and France. The statistical analysis of this variability has led to the use of complementary indices which quantify the circadian oviposition pattern, in particular the phase and the 
amplitude of the laying peaks. The first index, specific for the oviposition rhythm, compares the peaks occuring throughout scotophase and photophase. The second index, more objective and more efficient, is given by the first axis of a multivariate analysis, the correspondence analysis (reciprocal averaging). High levels of variability have been found within and between natural populations, however each population showed an oviposition rhythm which was characteristic and stable over the observed time period. Artificial selection which was applied to the French population brought about modifications in the oviposition pattern and led to two opposite phenotypes : a rhythm with a high peak at the beginning of the scotophase, and a rhythm with a maximum egg-laying during photophase. The variability of the peak amplitude at the onset of darkness is in part related to the daily fecundity and can be explained by the physiological capacity of females to retain mature ovocytes within the ovaries. In natural populations, the circadian oviposition rhythm could be maintained by natural selection acting on egg-laying behaviour.

Key words : Natural population, circadian rhythm, selection, multivariate analysis, D. melanogaster, isofemale lines.

\section{Introduction}

Les organismes sont soumis aux variations régulières, journalières et annuelles, de l'environnement ; et ceux qui ont pu synchroniser leur comportement et leur physiologie en réponse à ces variations naturelles ont accru leur chance de survie et leurs avantages sélectifs. C'est pourquoi, l'ensemble des fonctions vitales présente une rythmicité biologique dont l'étude (chronobiologie) aborde tous les niveaux depuis la biochimie jusqu'à l'écologie (BunNING, 1973 ; SAUNDERS, 1982). Ces hypothèses rendant compte de l'existence des rythmes biologiques reposent implicitement sur des fondements génétiques, en particulier sur la variabilité entre individus, qui jusqu'à présent n'ont guère été étudiés à la fois pour des raisons historiques et méthodologiques.

La majorité des travaux ont surtout cherché à mettre en évidence les mécanismes des rythmes aux niveaux moléculaire, cellulaire ou individuel. L'approche est extérieure à la Génétique et la variabilité observée, quelle que soit sa nature, est plutôt ignorée ou même passée sous silence (voir à ce sujet les préoccupations scientifiques développées lors du récent congrès de Chronobiologie de Seillac; Hugelin, 1982). En outre, les séries chronologiques sont longues et portent le plus souvent sur le même individu. Les mesures sont donc autocorrélées, ce qui provoque des difficultés statistiques qui s'ajoutent à celles plus générales du traitement de ces séries qui demandent des méthodes particulières pour déterminer la période ou les phases (voir DE PRins et al., 1977 ; Legendre \& Legendre, 1979).

Parmi les métazoaires, la Drosophile est une espèce remarquable car c'est la seule pour laquelle un ensemble d'informations génétiques relatives aux rythmes biologiques a été obtenu. En dehors des mutations affectant les rythmes d'émergence et d'activité (KONOPKA \& BENZER, 1971 ; KonOPKA, 1979), la variabilité génétique a pu être mise en évidence sur le rythme d'émergence. Dans ce cas, l'événement n'a lieu qu'une fois pendant la vie de l'organisme, et la variabilité entre les mesures traduit celle des individus. Par sélection artificielle, la phase de ce rythme a pu être modifiée (Pittendrigh, 1967 ; Clayton \& Paietta, 1972) sans toutefois affecter les mécanismes endogènes du rythme (PitTendrigh, 1967).

L'analyse de la variabilité des caractéristiques de phase et d'amplitude d'un autre comportement circadien de la drosophile a été entreprise sur le rythme d'oviposition. Cette variabilité a été recherchée entre familles au sein de différentes populations 
naturelles et en réalisant des expériences de sélection. Cette étude qui repose sur la technique des lignées isofemelles, habituellement utilisée en génétique des populations des drosophiles (David, 1979; Parsons, 1980), a nécessité l'application originale de deux méthodes complémentaires pour décrire et quantifier les rythmes, d'une part un indicateur empirique adapté à la forme des courbes de ponte, et d'autre part une analyse multidimensionnelle, l'analyse (factorielle) des correspondances (AFC).

\section{Matériel et méthodes}

\section{A. Populations de drosophiles}

Les drosophiles ont été capturées dans la nature juste avant les expériences dans deux localités : l'une en Afrique près de Brazzaville (R.P. du Congo), l'autre en France à Beynost (Ain) près de Lyon. Dans chacune des populations naturelles plusieurs prélèvements ont été effectués à un an d'intervalle, trois pour la population d'Afrique et deux pour la population de France. Après chaque prélèvement, une population a été fondée avec une centaine d'individus et, à la première ou à la deuxième génération d'élevage en masse au laboratoire (avant qu'une sélection ou une dérive aient pu se produire), cinq à quinze lignées isofemelles ont été constituées avec des couples pris au hasard. Les mesures du rythme de ponte ont porté sur les descendants (frères et sœurs) de ces différents couples.

\section{B. Elevages et expériences}

Les drosophiles utilisées pour l'expérimentation ont été élevées en faible densité de population, à $25^{\circ} \mathrm{C}$ sous photopériode LD $12: 12$, sur du milieu axénique composé de farine de maïs et de levure de bière. Après l'émergence, les adultes ont été maintenus en groupe d'environ cinquante avant d'être placés dans le dispositif de mesure décrit précédemment (David \& Foulllet, 1973 ; Allemand, 1983 a). Pendant l'expérience, les drosophiles (4 femelles et 4 mâles) sont placées dans une enceinte sous laquelle défile le substrat de ponte. L'emplacement des œufs sur le substrat (milieu axénique) permet de connaître le moment de la ponte. Le rythme a été mesuré pendant quatre jours consécutifs à $25^{\circ} \mathrm{C}$, sous photopériode LD 12:12 (photophase de $8 \mathrm{~h}$ à $20 \mathrm{~h}$ ), mais seuls les résultats du troisième et quatrième jour ont été pris en compte de façon à éviter les effets provoqués par la découverte d'un environnement nouveau (Allemand, 1983 a). L'éclairement d'environ 1100 lux a été fourni par des tubes fluorescents «lumière du jour»; les passages lumière-obscurité et l'inverse ont eu lieu brusquement, sans transition.

Par sélection de type familial (caractère mesuré uniquement chez les femelles), des lignées ont été suivies pendant 9 générations pour obtenir deux types de rythmes de forte amplitude, l'un avec un pic pendant la photophase, l'autre avec un pic en début de scotophase. A chaque génération de sélection, 10 couples ont été isolés et le rythme de leurs descendants a été mesuré. Les 2 familles présentant les meilleures caractéristiques ont été retenues, et les individus de ces 2 familles ont été mélangés. Les croisements ont eu lieu au hasard avec environ 40 reproducteurs, et les descendants ont servi à fonder les 10 familles de la génération suivante. 


\section{Traitement des données}

Deux méthodes originales permettant de caractériser globalement par la phase et l'amplitude des maximums un rythme de période connue ont été développées, l'une relativement empirique et adaptée spécifiquement aux caractéristiques du profil de ponte des drosophiles, l'autre de portée beaucoup plus générale faisant appel à l'analyse des correspondances.

- Indicateur de profil de rythme. Des travaux antérieurs (Allemand, 1977) ont montré que, sous photopériode LD12:12, un pic de ponte de Drosophila melanogaster s'observe en début de scotophase. Cependant, une ponte faible a lieu tout au long de la journée avec parfois un deuxième maximum d'amplitude variable pendant la photophase. L'indicateur de profil de rythme tient compte de ces 2 pics, et est défini à partir des courbes de ponte (nombre d'œufs/ $q$ / unité de temps) en ne tenant compte que des valeurs de ponte supérieures à la moyenne journalière. L'indicateur est égal à la différence entre les moyennes de ces valeurs observées pendant la scotophase et de celles observées pendant la photophase ; soit en utilisant les symboles de la figure $3 \mathrm{~A}$ :

$$
\text { indicateur }=\sum_{\mathrm{j}=1}^{\mathrm{n}_{\mathrm{d}}} \frac{\mathrm{d}_{\mathrm{j}}}{\mathrm{n}_{\mathrm{d}}}-\sum_{\mathrm{k}=1}^{\mathrm{n}_{\mathrm{l}}} \frac{\mathrm{l}_{\mathrm{k}}}{\mathrm{n}_{\mathrm{l}}}
$$

Cet indicateur a comme dimension un nombre d'œufs, et l'unité de temps choisie pour le calculer est égale à 2 heures. Si la ponte est totalement groupée sur 2 heures et en supposant qu'elle atteigne le maximum possible (environ 50 œufs), la valeur de l'indicateur sera de $-50 \mathrm{si}$ la ponte a lieu pendant la photophase et de +50 si elle a lieu pendant la scotophase. Les valeurs de l'indicateur seraient donc théoriquement comprises entre environ -50 et +50 ; toutefois, ces valeurs ne seront jamais atteintes car la ponte est toujours étalée sur plusieurs heures. L'indicateur est nul si les valeurs maximales de ponte sont également réparties entre la photophase et la scotophase.

- Analyse (factorielle) des correspondances (AFC). A l'issue d'une expérience, l'ensemble des données peut être traité globalement par une analyse multidimensionnelle : l'analyse des correspondances (BENZECRI, 1973 ; LEgENDRE \& LEgENDRE, 1979 ; GAUCH, 1982). Par cette méthode, particulièrement adaptée aux dénombrements, chaque courbe de ponte est représentée par une distribution de probabilités dans un espace à $n$ dimensions (n étant le nombre de mesures par cycle de 24 heures, le plus souvent ramené à 12) muni de la distance dite de $\chi^{2}$. L'inertie du nuage de points constitué par les courbes de ponte est analysée dans cet espace par rapport à son centre de gravité qui a pour coordonnées la distribution marginale calculée sur l'ensemble des courbes. Le but de l'analyse est de rechercher dans ce nuage de points les axes qui rendent compte de la plus grande variabilité. On définit ainsi un plan ou un sous-espace de faible dimension sur lequel on projette les points observés. Les coordonnées de chaque point dans ce sous-espace remplacent les courbes initiales. Elles permettent en particulier de construire des courbes ajustées qui reproduisent l'essentiel de la variabilité retenue.

L'échange des rôles entre heures et courbes de ponte conduit à une analyse du même type bien qu'a priori différente, qui fournit en fait les mêmes axes. Ceci justifie une représentation simultanée des heures et des courbes de ponte sur le même graphique. Les heures placées loin de l'origine contribuent à l'inertie restituée par le plan des axes considérés; d'autre part, les individus placés près de ces heures 
sont ceux qui sont concernés par le phénomène ainsi mis en évidence. On obtient de ce fait une visualisation directe de la signification des axes.

A l'issue de l'analyse, chaque courbe de ponte peut être caractérisée par des codages qui sont les coordonnées de cette courbe sur les axes principaux (le plus souvent deux): Contrairement à l'indicateur présenté précédemment, les codages fournis par l'analyse des correspondances sont définis de façon objective dans le contexte de l'étude effectuée. Cette méthode présente donc une portée générale et ne suppose aucune hypothèse préalable quant à la forme des courbes.

- En définitive, les méthodes utilisées pour quantifier les rythmes ont des conditions d'emploi différentes. Les valeurs d'une AFC sont définies dans le contexte d'une étude globale et sont donc relatives alors que l'indicateur de profil de rythme peut être calculé sur chacune des courbes indépendamment des autres, comme par exemple à chaque génération dans le cas des sélections.

\section{Résultats}

Plusieurs niveaux hiérarchiques de variabilité peuvent être définis : la population, le prélèvement et la famille. Chaque famille est mesurée pendant deux jours qui peuvent être considérés comme des répétitions. Le niveau individu n'a pas été considéré ici car l'expression du rythme de ponte est fonction du groupement qui synchronise les individus (Allemand, 1983 b).

\section{A. Description de la variabilité}

\section{Analyse qualitative des courbes de ponte}

A titre d'exemple, le rythme de ponte de quatre lignées prises dans chacune des deux populations est présenté à la figure 1. Pour la population d'Afrique, le rythme présente dans tous les cas un maximum de ponte placé au début de la scotophase. Les différences entre lignées portent sur la position du pic de ponte qui peut être à la première ou à la deuxième heure d'obscurité, sur son amplitude et aussi sur l'existence de pics secondaires pendant la photophase. De la même façon, la variabilité entre familles issues d'une population française porte essentiellement sur les taux relatifs de la ponte pendant la photophase et le début de l'obscurité.

L'analyse de l'ensemble des courbes caractérisant la variabilité de chaque population révèle que les deux populations ont des rythmes similaires mais que d'une façon générale la population africaine montre des maximums d'amplitude plus élevée que ceux de la population française. En outre, les rythmes de ponte conservent d'un jour à l'autre leurs caractéristiques. Ces conclusions apparaissent plus clairement sur des courbes moyennes de rythme (fig. 2) qui sont calculées sur l'ensemble des familles d'un même prélèvement dans une même population et qui font disparaître la variabilité interfamiliale. Ces courbes montrent également que les rythmes sont semblables d'un prélèvement à l'autre au sein d'une même population. 

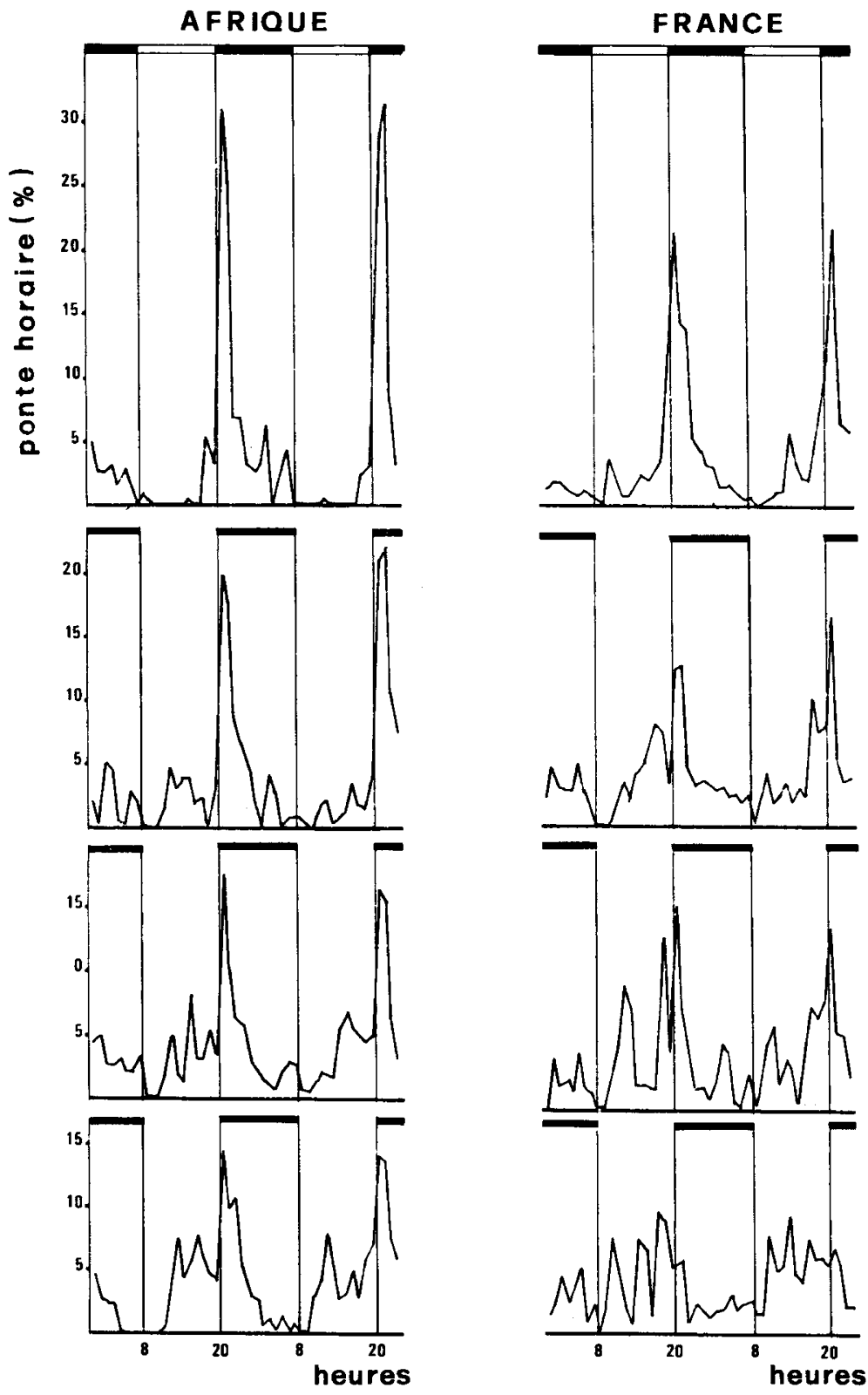

FIG. 1

Variabilité du rythme circadien de ponte de $\mathrm{D}$. melanogaster.

Variability of the circadian oviposition rhythm of D. melanogaster.

Exemples de rythmes mesurés sur différentes familles issues de deux populations naturelles (Afrique et France). Chaque famille est étudiée pendant deux jours successifs à $25^{\circ} \mathrm{C}$ sous une photopériode LD $12: 12$.

Different egg laying patterns of various families from 2 natural populations (tropical Africa and France). Each family was studied during two successive days, at $25^{\circ} \mathrm{C}$ under LD $12: 12$ photoperiod. 

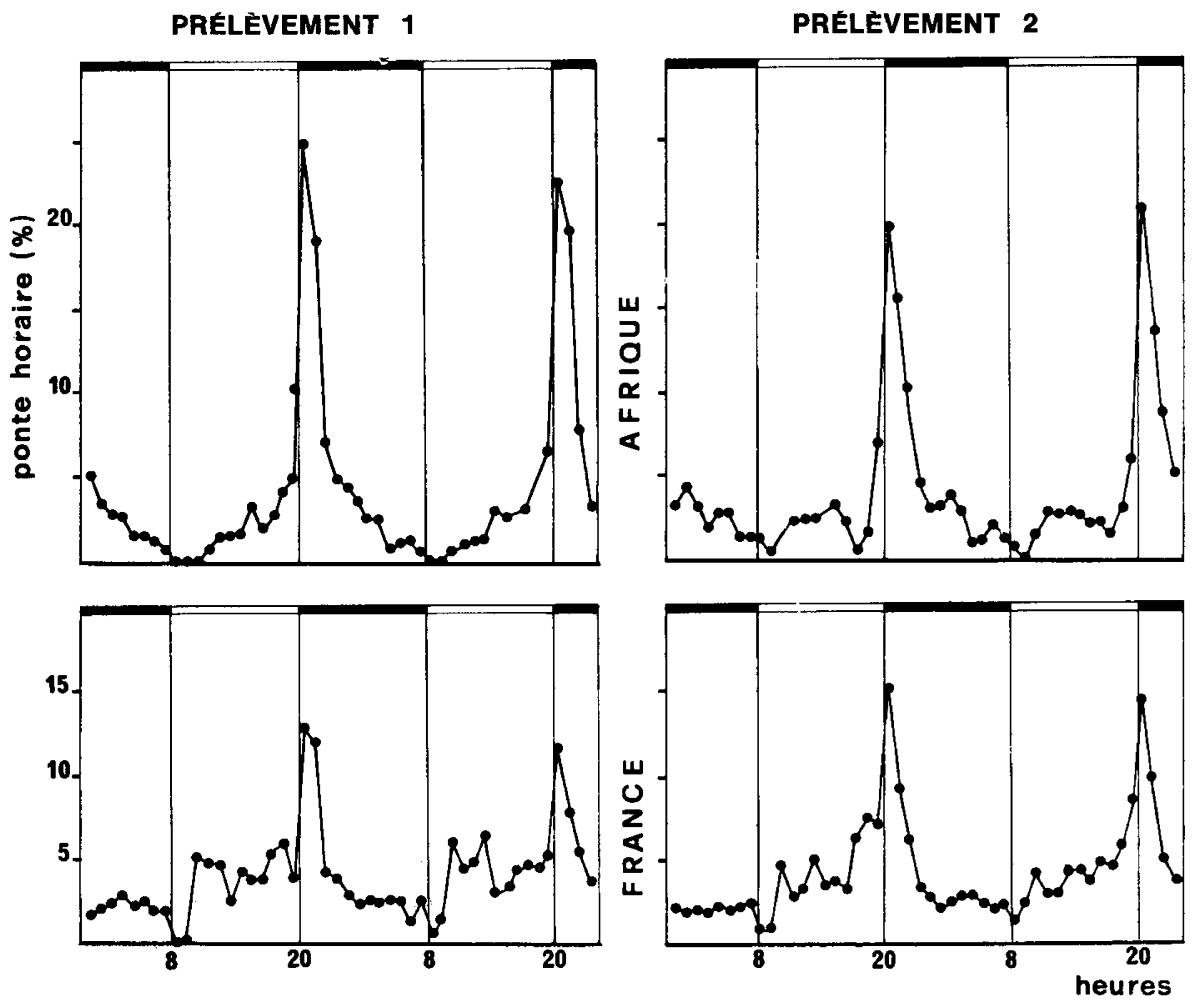

FIG. 2

Courbes moyennes de rythmes sous photopériode LD $12: 12$ pour deux prélèvements effectués dans les deux populations naturelles.

Average curves of oviposition rhythm ot two samplings from two natural populations.

Nombre de familles étudiées : Afrique, prélèvement 1:7, prélèvement $2: 5$. France, prélèvement $1: 10$, prélèvement $2: 5$.

LD 12:12 photoperiod; number of families : Africa, sampling $1: 7$, sampling $2: 5$; France, sampling $1: 10$, sampling $2: 5$.

\section{Analyse quantitative à l'aide des indicateurs}

A partir des données brutes (nombres d'œufs par relevé, toutes les deux heures), la variabilité a été décrite en utilisant les indicateurs définis précédemment, de façon à permettre une analyse statistique ultérieure.

- Indicateur de profil de rythme (i)

La distribution des indices calculés pour chaque population suit une loi normale (fig. 3 B). Entre populations, les distributions sont de même forme (variances non différentes) mais les moyennes sont très différentes : Afrique 7,88 $\pm 0,52$, France $4,54 \pm 0,84, \mathrm{t}=3,57, \mathrm{p}<0,001$. La valeur supérieure de l'indicateur pour la population africaine traduit une ponte préférentielle plus forte pendant la scotophase, ce qui confirme les observations qualitatives précédentes des figures 1 et 2 . 

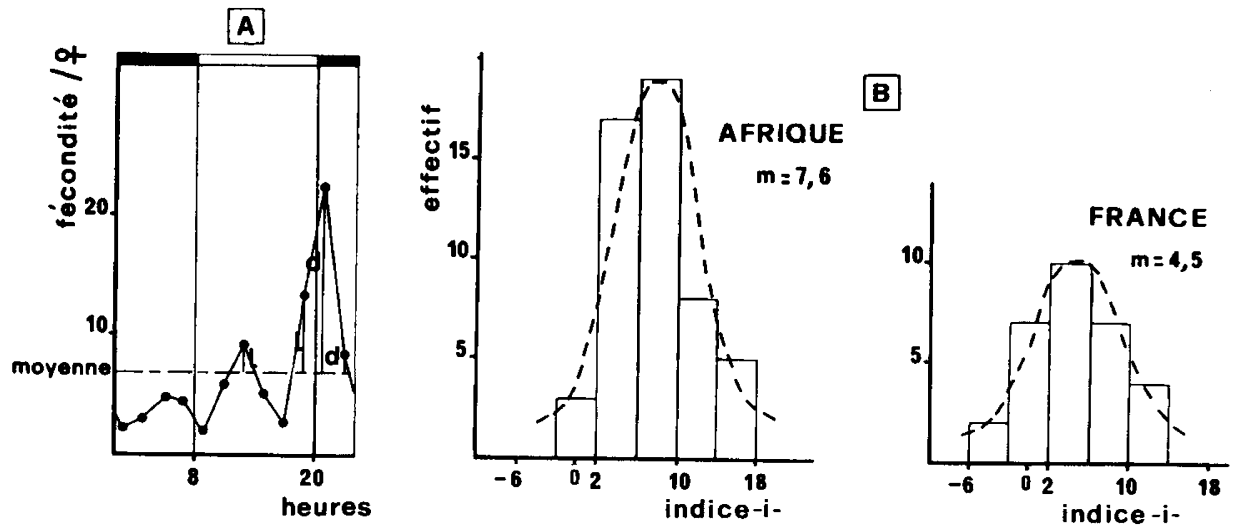

FIG. 3

A

Mode de calcul de l'indicateur $i$ utilisé pour quantifier le rythme de ponte sous photopériode LD $12: 12$.

Calculation method of the $i$ index used to quantify the oviposition rhythm under LD 12:12 photoperiod.

La fécondité (nombre d'œufs) est mesurée toutes les deux heures et seules les valeurs supérieures à la moyenne bihoraire journalière sont prises en compte. L'indicateur est la différence entre les moyennes de ces valeurs pendant l'obscurité (d) et celles des valeurs pendant l'éclairement (l). Pour cet exemple $\mathrm{i}=3,84$.

$$
\text { Indice }=\sum_{j=1}^{n_{d l}} \frac{d_{j}}{n_{l}}-\sum_{k=1}^{n_{l}} \frac{1_{k}}{n_{l}}
$$

The fecundity (number of eggs) is checked every 2 hours, and only the values higher than the daily bihourly average are retained. The index is the difference between the means of the significant values scored during the darkness $(d)$ and during the light period (l). In this case $i=3.84$.

$$
\text { Index }=\sum_{j=1}^{n_{l}} \frac{d_{j}}{n_{d}}-\sum_{k=1}^{n_{l}} \frac{l_{k}}{n_{l}}
$$

Histogramme des valeurs de $i$ dans les deux populations étudiées.

Histogram of the $i$ index values within the two populations.

Dans les deux cas les distributions peuvent être assimilées à une courbe de Gauss : Afrique : $\mathrm{i}=7,88 \pm 0,52$ ( $\left.\mathrm{n}=52, \chi^{2}=3,40,2 \mathrm{ddl}\right)$;

France : $\mathrm{i}=4,54 \pm 0,84$ ( $\left.\mathrm{n}=30, \chi^{2}=0,26,2 \mathrm{ddl}\right)$.

In each case, the distribution can be fitted to normal distribution :

Africa : $i=7.88 \pm 0.52\left(n=52, \chi^{3}=3.40, f d 2\right)$;

France : $i=4.54 \pm 0.84\left(n=30, \chi^{3}=0.26, f d 2\right)$. 


\section{- Analyse des correspondances (AFC)}

A l'issue d'une analyse globale des données, le cycle photopériodique (heures) et les courbes de ponte sont portées sur le même plan défini par les deux premiers facteurs de l'analyse (fig. 4). Le premier facteur (45,2 p. 100 de la variabilité) oppose les premières heures de l'obscurité à l'ensemble des autres heures et représente ainsi l'amplitude relative du pic de ponte au début de la scotophase. Le deuxième facteur $(10,3$ p. 100 de la variabilité) oppose le début au milieu de la photophase et rend compte ainsi de l'amplitude et de la phase des maximums observés pendant la photophase. Le troisième facteur (non représenté, 9,3 p. 100 de la variabilité) traduit les variations du taux de ponte pendant les toutes premières heures de la photophase.

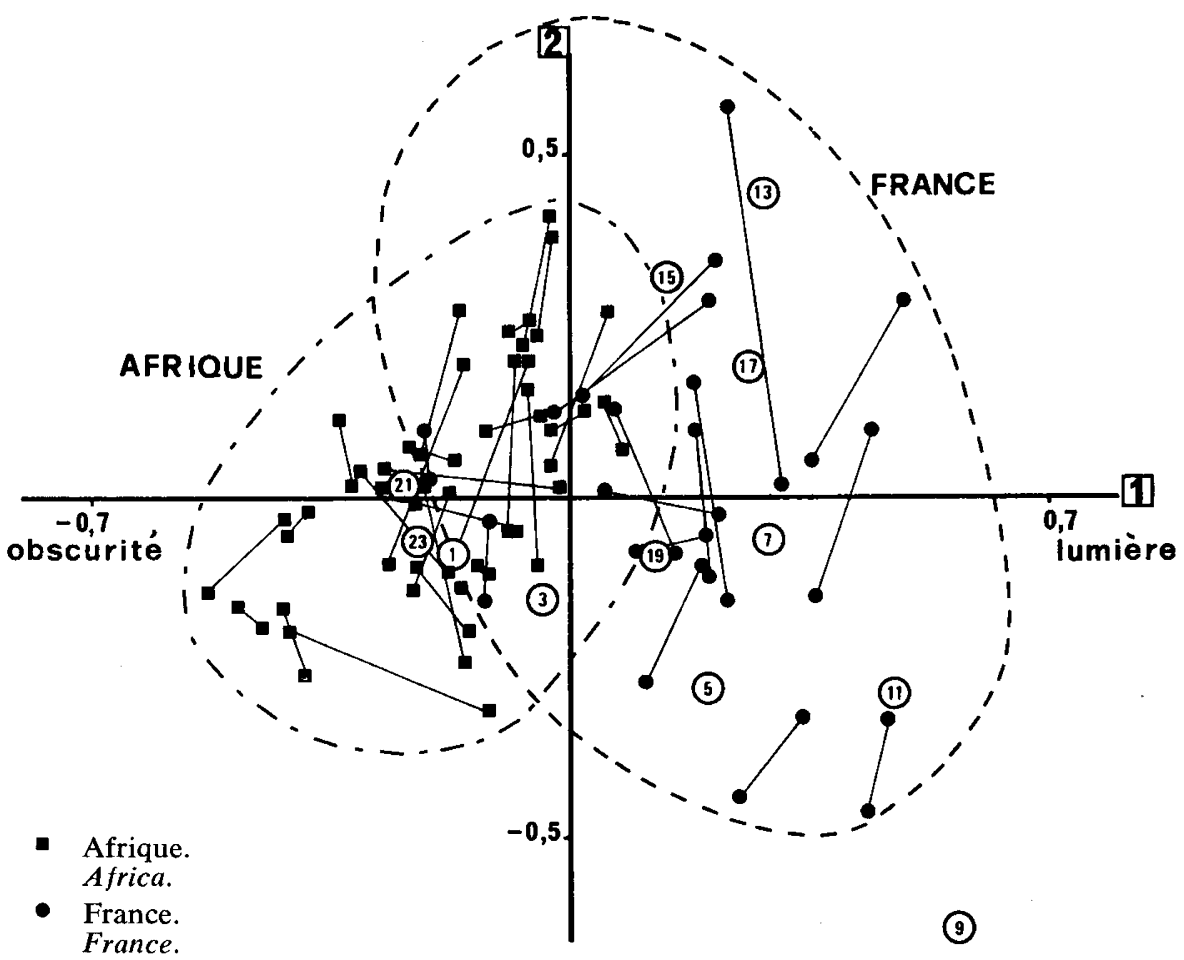

Fig. 4

Représentation de la variabilité du rythme de ponte

après transformation des courbes journalières par une analyse des correspondances (AFC). Variability of the oviposition rhythm after transformation of the daily egg laying curves by a correspondence analysis (reciprocal averaging; GAUCH, 1982).

Dans le plan défini par le $1^{\text {er }}$ et le $2^{\circ}$ facteur de $1^{\prime} A F C$ sont portées à la fois les représentations des courbes de ponte et du cycle photopériodique LD 12:12 (obscurité de $20 \mathrm{~h}$ à $8 \mathrm{~h}$ ). Les nombres encerclés correspondent aux heures et les couples de points réunis par une droite correspondent aux deux mesures d'une même famille.

The daily curves and light cycle LD 12:12 (hours are circled, darkness from 20 to 8 h) are plotted on the same diagram (1st axis corresponding to the night peak amplitude, 2nd axis corresponding mainly to the egg laying distribution during the photophase). The pairs of joined points are the two measures of the same family. 
Sur le plan des deux premiers facteurs, les mesures des deux populations sont discriminées par le premier facteur de part et d'autre de l'origine. Cette observation est confirmée par le test de Wilks (Dagnelie, $1975 ; \chi^{2}=58,5,2 \mathrm{ddl}, \mathrm{p}<0,001$ ).

\section{B. Sources de variabilité}

Chaque courbe de ponte peut être définie à l'aide de l'indicateur i ou des coordonnées sur les axes principaux de l'AFC. Ces paramètres visent à définir le même phénomène biologique et doivent avoir des points communs que l'on peut mettre en évidence par leur corrélation. Seule la corrélation entre i et le premier facteur de l'AFC est significative $(r=|0,65|, n=82, p<0,001)$ alors que les corrélations entre $\mathrm{i}$ et les autres facteurs importants de l'AFC ne sont pas différentes de zéro.

La signification des différentes sources de variabilité : interpopulation, intrapopulation (interprélèvement), interfamille (intraprélèvement) et interjour (intrafamille) a été analysée sur l'ensemble des résultats par des analyses de variance hiérarchiques portant à la fois sur l'indicateur i et le premier facteur de l'AFC (tabl. 1). Les résultats de ces deux analyses sont similaires, toutefois l'analyse portant sur le premier facteur de l'AFC est systématiquement plus sensible, ce qui montre que cette méthode est plus performante pour ce genre d'étude.

\section{Tableau 1}

Sources de variabilité du rythme circadien de ponte chez D. melanogaster.

Origin of the variability of the circadian oviposition rhythm of D. melanogaster.

\begin{tabular}{|c|c|c|c|c|c|c|}
\hline \multirow{2}{*}{ Origine } & \multirow{2}{*}{ Source de variabilité } & \multirow{2}{*}{ ddl } & \multicolumn{2}{|c|}{ Indicateur } & \multicolumn{2}{|c|}{$1^{\text {er }}$ facteur AFC } \\
\hline & & & Variance & $\mathbf{F}$ & Variance & $\mathbf{F}$ \\
\hline \multirow{2}{*}{ Totale } & $\begin{array}{l}\text { Interfamille } \ldots \ldots \ldots \\
\text { Interprélèvement } \ldots \ldots \\
\text { Interpopulation } \ldots \ldots \\
\text { Résiduelle } \ldots \ldots \ldots\end{array}$ & $\begin{array}{r}36 \\
3 \\
1 \\
41\end{array}$ & $\begin{array}{r}23,776 \\
7,508 \\
211,469 \\
11,088\end{array}$ & $\begin{array}{c}2,14^{*} \\
<1 \\
19,07^{* *}\end{array}$ & $\begin{array}{l}0,0531 \\
0,0353 \\
2,3317 \\
0,0060\end{array}$ & $\begin{array}{c}8,53 * * \\
<1 \\
43,90 * *\end{array}$ \\
\hline & $\begin{array}{l}\text { Interjour } \ldots \ldots \ldots \\
\text { Interfamille } \ldots \ldots \ldots \\
\text { Interaction } \ldots \ldots \ldots\end{array}$ & $\begin{array}{r}1 \\
40 \\
40\end{array}$ & $\begin{array}{r}0,333 \\
27,242 \\
11,362 \\
\end{array}$ & $<1$ & $\begin{array}{l}0,0028 \\
0,1088 \\
0,0061\end{array}$ & $<1$ \\
\hline Afrique & $\begin{array}{l}\text { Interfamille } \ldots \ldots \ldots \\
\text { Interprélèvement } \ldots \ldots \\
\text { Résiduelle } \ldots \ldots \ldots \\
\text { Coefficient intraclasse }\end{array}$ & $\begin{array}{r}23 \\
2 \\
26\end{array}$ & $\begin{array}{r}16,520 \\
9,942 \\
12,491 \\
0\end{array}$ & $\begin{array}{l}1,32 \\
<1\end{array}$ & $\begin{array}{l}0,0428 \\
0,0458 \\
0,0058\end{array}$ & $\begin{array}{l}7,38^{* *} \\
1,08\end{array}$ \\
\hline France & $\begin{array}{l}\text { Interfamille } \ldots \ldots \ldots \\
\text { Interprélèvement } \ldots \ldots \\
\text { Résiduelle } \ldots \ldots \ldots \\
\text { Coefficient intraclasse }\end{array}$ & $\begin{array}{r}13 \\
1 \\
15\end{array}$ & $\begin{array}{r}36,614 \\
2,639 \\
8,656 \\
0\end{array}$ & $\begin{array}{c}4,23 * \\
<1\end{array}$ & $\begin{array}{l}0,0713 \\
0,0142 \\
0,0064\end{array}$ & $\begin{array}{c}11,15^{* *} \\
<1\end{array}$ \\
\hline
\end{tabular}

Analyses de variances hiérarchiques portant sur les valeurs des indicateurs servant à quantifier les rythmes (indicateur $\mathrm{i}$ et $1^{\mathrm{er}}$ facteur AFC). Les coefficients de corrélation intraclasse représentent la stabilité du rythme de ponte d'une même famille. Effets significatifs : * $\mathrm{p}<0,01 ; * k^{*} \mathrm{p}<0,001$. Results of hierarchical analysis of variance on the indices used to quantify the rhythms ( $i$ index and 1st factor of the reciprocal averaging). The correlation coefficients intraclass show the stability of the oviposition rhythm within families. Significant effects : * $p<0.01 ; * * p<0.001$. 
Les analyses de variance confirment les observations précédentes, en particulier, la différence globale entre les deux populations et révèlent également les différences entre lignées. Elles montrent que le rythme est stable pendant l'expérimentation, surtout pour la population française (coefficient de corrélation intraclasse, tabl. 1), et que les prélèvements successifs dans une même population ont des caractéristiques identiques. En conclusion, le rythme de ponte constitue donc une caractéristique propre à chaque population, stable en moyenne au cours du temps mais présentant une certaine variabilité intrapopulation.

\section{Nature génétique de la variabilité : sélection du rythme}

Les conditions standardisées de laboratoire suppriment la plus grande part de la variabilité environnementale et la méthode des lignes isofemelles permet de révéler la variabilité génétique intrapopulation. Une expérience de sélection réalisée à partir de la population française a permis de confirmer et de préciser les bases génétiques de cette variabilité.

A partir de cette population (fig. $5 \mathrm{~A}$ ), des lignées ont été sélectionnées en utilisant l'indicateur $\mathrm{i}$ pendant 9 générations pour obtenir deux types de rythmes, l'un avec un pic pendant la photophase et l'autre avec un pic en début de scotophase. Le rythme de ponte a répondu à la sélection et l'indicateur de rythme a varié au cours des générations (fig. $5 \mathrm{~B}$ ). Après la $5^{\mathrm{e}}$ génération des plateaux ont été atteints et les pressions de sélection sont restées sans effet. La sélection a conduit à des phénotypes très différents à la fois en phase et en amplitude (fig. $5 \mathrm{C}$ et $\mathrm{D}$ ). Dans le cas d'un pic à l'obscurité $(i=8,9)$ la ponte pendant les deux premières heures d'obscurité représente plus de 40 p. 100 de la ponte totale ce qui est semblable à ce qui peut être observé dans les populations naturelles les plus rythmées. La sélection pour un pic pendant la photophase a conduit à un phénotype dont le pic de ponte n'est pas très élevé mais plus étalé. Ce type de rythme est voisin des phénotypes obtenus après dérive génétique (Allemand \& DAvid, 1984) et est caractérisé par l'absence de pic de nuit et par des valeurs moyennes d'indicateurs négatives $(i=-1,5)$ qui ne sont jamais observées dans des populations naturelles.

Des croisements entre lignées sélectionnées (sélection basse, $\mathrm{sb}=$ pic pendant la photophase, $\mathrm{i}=-2,6$; sélection haute, $\mathrm{sh}=$ pic pendant la scotophase, $\mathrm{i}=10,7$ ), réalisés après la sixième génération de sélection donnent des descendants $F_{1}$ et $F_{2}$ dont les rythmes sont intermédiaires mais proches des parents à forte ponte pendant la photophase. Dans le cas des croisements femelle sb par mâle sh $(\mathrm{i}=-2,6 \times \mathrm{i}=$ 10,7), les descendants ont les indicateurs suivants: $F_{1}=-1,7 ; F_{2}=1,4$. Dans l'autre sens (femelle sh par mâle sb), les indicateurs sont $F_{1}=0,2$ et $F_{2}=-2,1$. Les variations des valeurs des indices entre $F_{1}$ et $F_{2}$ paraissent traduire un léger effet du chromosome $\mathrm{X}$ (grand-maternel à l'état homozygote à la $\mathrm{F}_{\mathrm{g}}$ ). Une analyse plus approfondie des déterminismes génétiques impliqués fera l'objet d'un travail ultérieur.

\section{Relation entre la variabilité du rythme de ponte et celle de la fécondité journalière}

Lors de l'analyse des profils de rythme, le critère étudié est la distribution du nombre d'œufs au cours des différentes tranches horaires. Le nombre total d'œufs pondus par jour est lui-même soumis à une variation génétique reposant essentielle- 
A
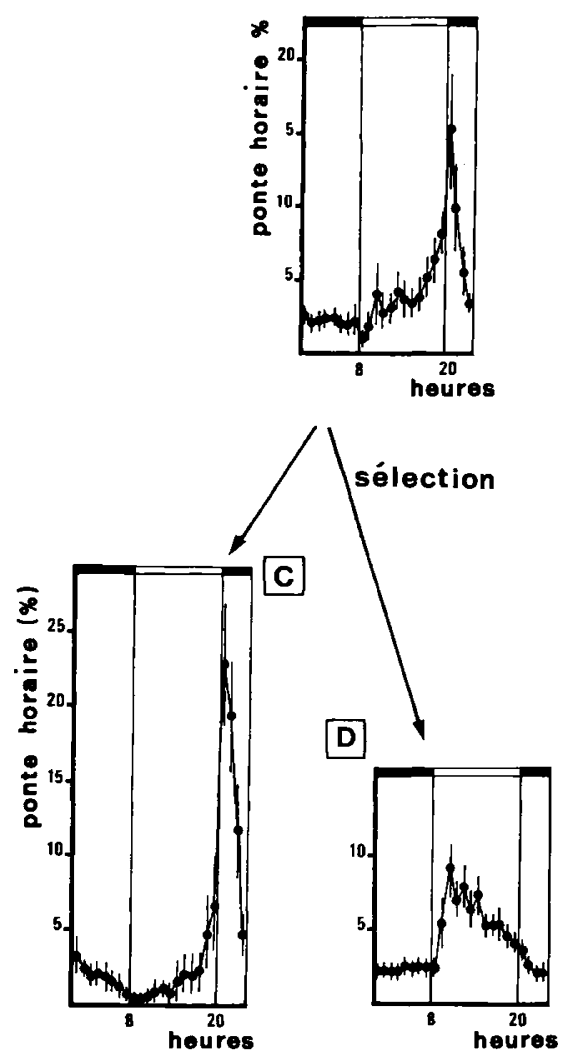

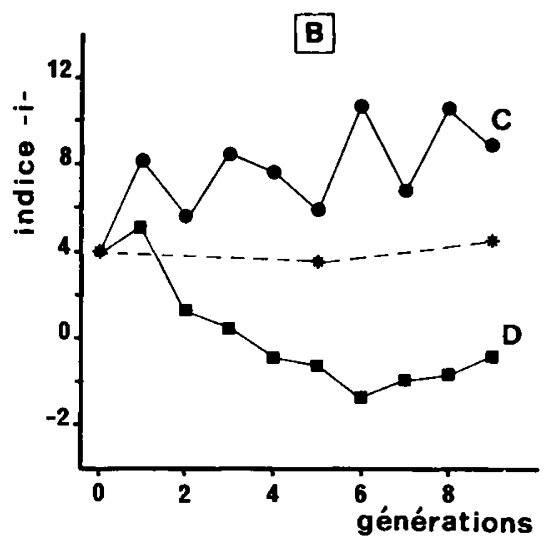

E

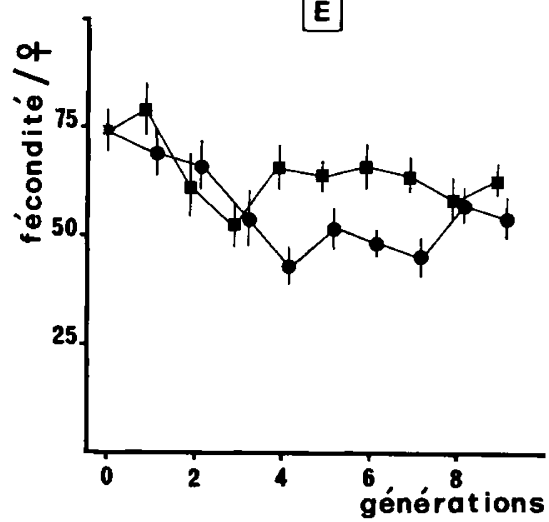

FIG. 5

Sélection artificielle du rythme circadien de ponte de $\mathrm{D}$. melanogaster. Selection of the circadian oviposition rhythm of D. melanogaster.

\section{A}

Rythme moyen de ponte de la population naturelle d'origine (France) mesuré sous photopériode LD 12:12.

Average curve of the laying pattern under LD 12:12 of the natural population before selection.

B

Evolution du profil de rythme au cours de la sélection mesurée par l'indicateur $i$.

Response to the selection (expressed with the $i$ index).

- Sélection pour un rythme avec une ponte forte en début d'obscurité.

Selection of a rhythm pattern with high egg laying at the onset of darkness.

- Sélection pour un rythme avec une forte ponte pendant la photophase.

Selection of a rhythm pattern with high egg laying during photophase.

$$
\text { C - D }
$$

Rythmes moyens de ponte des lignées après 9 générations de sélection. Average curves of the laying pattern after 9 generations.

E

Evolution de la fécondité journalière (nombre d'œufs/q/jour) au cours de la sélection. Mêmes symboles que $B$.

Evolution of the daily fecundity (number of eggs/ $q /$ day) during the selection experiment. 
ment sur celle du nombre d'ovarioles (voir David, 1970 ; Bouletreau-Merle et al., 1982). Les relations éventuelles entre les caractéristiques du rythme et la fécondité journalière ont été recherchées au sein des 2 populations.

Les drosophiles d'origine africaine pondent moins que celles d'origine française et présentent un pic de ponte plus élevé $(58,4 \pm 3,1$ œufs/ $q$ /jour contre 77,6 $\pm 5,1$; $\mathrm{t}=6,8, \mathrm{p}<0,001)$. Cette corrélation négative est retrouvée dans l'évolution de la fécondité lors des expériences de sélection. Après une diminution globale de la fécondité due probablement à la dépression consanguine, la fécondité s'est stabilisée à un niveau inférieur pour les lignées ayant un pic de forte amplitude au début de la scotophase (fig. $5 \mathrm{E}$ ). La relation inverse entre amplitude du pic de ponte en début d'obscurité et la fécondité journalière a aussi été recherchée au sein des 2 populations en utilisant successivement les 2 indicateurs.

Les corrélations entre la fécondité et les indicateurs ont été calculées pour chacune des populations et sont présentées figure 6 . La corrélation n'est significative
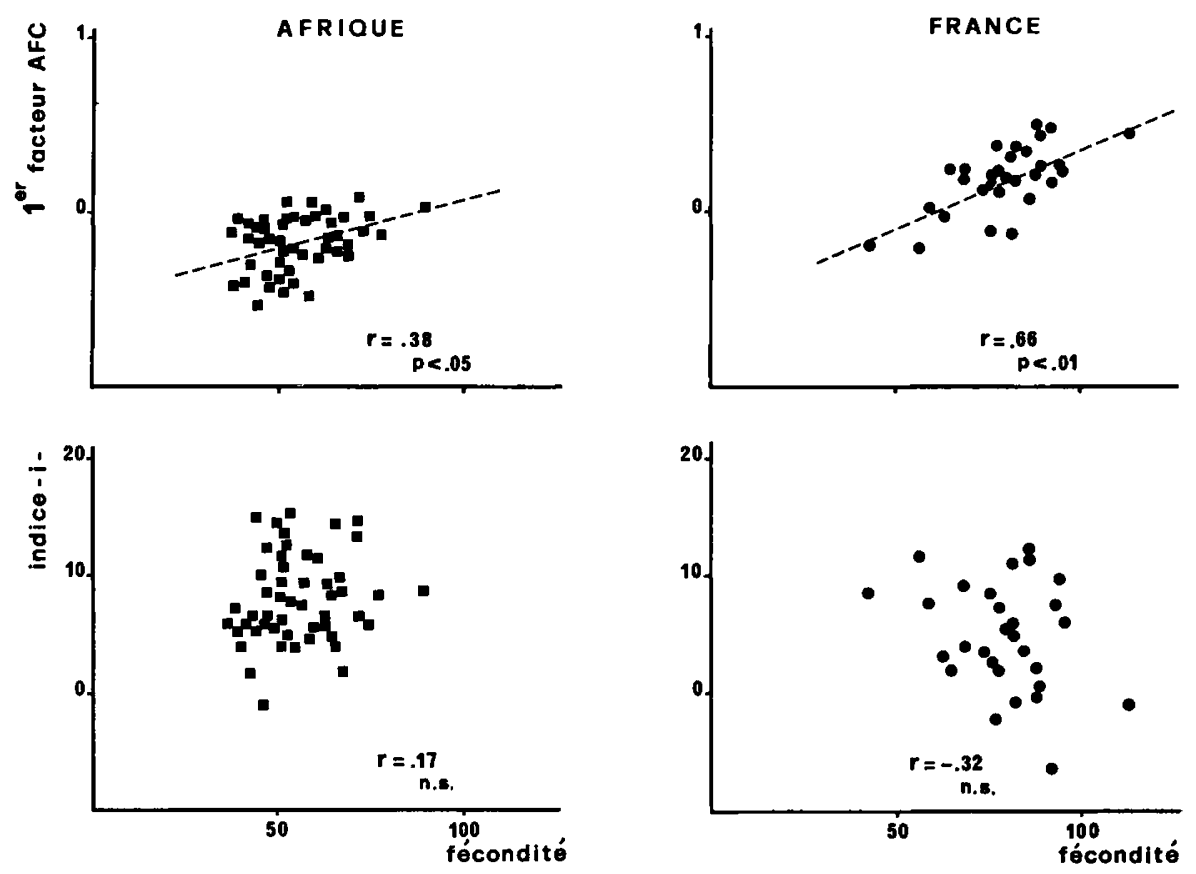

FIG. 6

Relation entre la variabilité du rythme de ponte et celle de la fécondité journalière au sein de chaque population.

Relation between the variability of the oviposition rhythm and the variability of the daily fecundity (number of eggs/day) within each population.

Corrélations entre les indicateurs quantifiant les rythmes (indicateur $i$ et premier facteur $\mathrm{AFC})$ et la fécondité journalière. Afrique, $n=52$; France, $n=30 . r$ : coefficient de corrélation linéaire, n.s. : non significatif.

Correlations between the indices used to quantify the rhythm patterns ( $i$ index and first factor of the reciprocal averaging) and the daily fecundity. Africa : $n=52$; France : $n=30 . r$ : correlation coefficient, n.s. : non significant. 
que pour le premier facteur de l'AFC ce qui conduit à préciser la signification biologique des indicateurs. Le premier facteur de l'AFC quantifie essentiellement l'importance relative du pic de ponte à l'obscurité (ponte de 20 à 24 heures) et est donc en étroite relation avec le nombre d'œufs pondus, alors que l'indicateur i est plus global puisqu'il compare les pontes pendant la scotophase et la photophase.

En définitive, il n'y a pas de relation très étroite entre rythme et fécondité journalière, toutefois l'amplitude relative des pics peut montrer une relation inverse avec la fécondité journalière.

\section{Discussion - Conclusion}

Peu de travaux ont été consacrés à des études génétiques de la variabilité des rythmes biologiques. Une telle variabilité est parfois mentionnée entre espèces, par exemple chez les drosophiles (Hardeland \& Stange, 1973; Allemand, 1976 a) ou entre individus comme chez Periplaneta americana (RIVAulT, 1981) ou bien chez Triturus alpestris (MARTIN, 1982) où la variabilité individuelle se manifeste à la fois sur la phase et la période du rythme. Chez $D$. melanogaster, la variabilité du rythme de ponte porte sur la phase et l'amplitude des maximums et apparaît à plusieurs niveaux, entre populations d'origine différente et entre familles issues d'une même population. Le support de cette variabilité et ses implications génétiques et adaptatives seront discutés et interprétés après avoir tiré quelques conclusions sur les méthodes pour quantifier les rythmes.

L'analyse de la variabilité génétique des rythmes se heurte à des difficultés pratiques provenant de la nature des données, qui sont nombreuses et rarement indépendantes. C'est pourquoi, des paramètres qui rendent compte à la fois de la phase et de l'amplitude des maximums ont été définis. Considérant que la période du phénomène est connue ( 24 heures, cycle LD $12: 12$ ), l'indicateur de profil de rythme $\mathrm{i}$ défini par les pics de ponte pendant la scotophase et la photophase est bien adapté pour quantifier et comparer des courbes de ponte et également pour révéler la variabilité. Cet indicateur, indépendant de la ponte journalière (cf. fig. 6), est todtefois empirique et subjectif, ce qui en limite les possibilités d'utilisation. Au contraire, l'analyse des correspondances apparaît comme une technique particulièrement performante, d'une part pour décrire la forme des courbes de ponte par rapport au cycle photopériodique, d'autre part pour analyser globalement plusieurs profils et les classer objectivement entre eux dans l'espace temps. En ne conservant à l'issue d'une telle analyse que les coordonnées du $1^{\text {er }}$ facteur qui traduit l'amplitude relative du pic de ponte principal, des profils de rythme peuvent être comparés par des méthodes statistiques simples. Cette approche, qui a permis de mettre en évidence différents niveaux de variabilité dans les populations devrait pouvoir s'appliquer à de nombreux autres exemples de rythmes biologiques et, en particulier, permettre des comparaisons entre espèces différentes. Le nouveau domaine d'application de cette méthode d'analyse accroît l'intérêt de l'AFC qui semble actuellement s'ouvrir à des champs d'application très larges comme l'écologie (Gloagen \& Gautier, 1981 ; Chessel et al., 1982) ou à la génétique (Grantham et al., 1981; Fondevilla et al., 1982).

Le rythme de ponte repose sur deux phénomènes interdépendants, le fonctionnement ovarien conduisant à la formation des œufs et les mécanismes (surtout compor- 
tementaux) modulant le dépôt des œufs. Pendant la photophase, la production ovarienne de $D$. melanogaster est très forte pour chacune des populations (Allemand, $1976 \mathrm{~b}$ et résultats non publiés); les ovocytes mûrs peuvent être pondus sans délai ou bien conservés en rétention dans les ovaires. Cette rétention qui permet d'alimenter le pic de ponte en début d'obscurité, est la conséquence du blocage de l'oviposition en réponse à des signaux de l'environnement comme par exemple l'éclairement, le substrat ou le groupement (voir Allemand, 1983 a ; 1983 b). La variabilité peut donc avoir une double origine portant sur le fonctionnement ovarien ou sur l'oviposition.

Le nombre de tubes ovariens détermine en partie la fécondité journalière. Ce caractère présente une forte variabilité génétique intrapopulation (DAvID, 1979; Bouletreau-Merle et al., 1982) qui est en partie à l'origine de la variabilité de la production ovocytaire. Suivant le nombre d'ovocytes formés, la rétention provoque une contrainte physiologique (distension de l'abdomen) d'autant plus forte que la production journalière est plus élevée. Ce phénomène explique la relation négative entre fécondité et l'amplitude relative du pic de ponte à l'obscurité révélée par le premier facteur de l'AFC. Cette relation qui est à l'origine d'une partie de la variabilité, complète le modèle fonctionnel développé précédemment (Allemand, $1983 \mathrm{a} ; 1983$ b) montrant que le profil de rythme est déterminé par des facteurs exogènes qui modulent la rétention d'ovocytes. La production ovocytaire constitue un facteur particulier qui est interne et propre à l'individu. Cependant cette relation ne peut expliquer à elle seule l'évolution des courbes de rythme pendant l'expérience de sélection. Entre les deux lignées sélectionnées, les fécondités restent assez proches bien que statistiquement différentes, par contre les rythmes sont extrêmement différents (phases opposées). Dans le cas de la lignée montrant un maximum de ponte pendant la photophase, le rythme de ponte suit celui de la production d'ovocytes (Allemand, 1976 b) et par conséquent la sélection a modifié la sensibilité aux agents extérieurs et/ou les mécanismes de régulation de l'oviposition, c'est-à-dire en terme de chronobiologie, le couplage entre le rythme endogène et son expression. Ce résultat est semblable à celui observé par PitTEndrigh (1967) à la suite de la sélection du rythme d'émergence. La sensibilité et la réponse aux divers facteurs environnementaux agissant sur l'expression du rythme (lumière, substrat, groupement) sont sous contrôle génétique comme l'ont montré indirectement des travaux sur la réponse phototactique (KAWANISHI \& WATANABE, 1978).

Le rythme circadien de ponte présente des différences significatives entre les populations étudiées malgré la variabilité intrapopulation. Celui de la population tropicale africaine présente un pic d'amplitude plus forte que celui de la population française, cette dernière ayant un fort taux de ponte pendant la photophase. Ces résultats confirment l'existence d'une variation clinale du rythme avec la latitude (Allemand \& David, 1976) où les valeurs moyennes entre populations d'origine différente sont les suivantes (exprimées par l'indicateur i) : Afrique tropicale $=7,4$, Afrique du Nord $=5,1$, France $=4,6$, Scandinavie $=2,9$. Ces différences sont renforcées par la stabilité du rythme entre les prélèvements successifs dans les mêmes populations naturelles. On peut donc en conclure que, au sein de chacune des populations, le rythme de ponte moyen est stable dans le temps et possède des caractéristiques génétiquement définies. Ce profil moyen doit résulter des pressions sélectives stabilisantes qui s'exercent sur la variabilité intrapopulation. Ce phénomène classique pour de nombreux caractères quantitatifs (taille, poids...) ou bien biochimiques (par exemple polymorphisme enzymatique) manque d'illustrations en chronobiologie. En effet, outre 
la complexité des phénomènes étudiés (dans ce cas fécondité et comportement d'oviposition), la notion de rythme fait appel à une dimension temporelle qui se retrouve dans l'expression des facteurs sélectifs, eux-mêmes cycliques.

L'hypothèse selon laquelle le phénotype moyen de la population est stabilisé par les pressions sélectives est confirmée par les résultats antérieurs obtenus après dérive génétique, montrant que les caractéristiques de phase et d'amplitude du rythme peuvent être perdues dans certaines sous-populations conservées pendant de nombreuses générations au laboratoire (Allemand \& DAvid, 1984). En outre la variabilité donne prise à la sélection, ce qui conduit à des phénotypes très différents en phase et en amplitude. Le rythme obtenu chez une population d'origine française après sélection pour un pic à l'obscurité est proche de celui observé spontanément chez certaines populations d'Afrique tropicale. Par contre celui avec un maximum pendant la photophase n'a pas d'équivalent dans la nature, ce phénotype faisant sans doute l'objet dans les. populations naturelles d'une contre-sélection.

Compte tenu des mécanismes physiologiques mis en jeu, la modulation du rythme par de nombreux facteurs exogènes de nature diverse et relativement indépendants (photopériode, habitat, relations biotiques) soumet les drosophiles à des pressions sélectives concourantes ou contradictoires, bien que toutes centrées sur la capacité de rétention et d'ovulation. Par exemple, un éclairement faible et un substrat favorable peuvent favoriser la ponte pendant la photophase mais la présence de nombreux autres individus retarde la ponte qui a lieu alors au début de la scotophase (Allemand, 1983 b). Dans l'hypothèse où le moment de la ponte a une valeur sélective en abaissant les taux de compétition, de prédation ou de parasitisme, ces pressions contradictoires doivent permettre un maintien de la variabilité génétique au sein de la population.

Reçu le 24 janvier 1983.

Accepté le 26 août 1983.

\section{Remerciements}

Nous remercions MM. et $\mathbf{M}^{\text {mes }} \mathbf{J}$. BoulÉtreau-Merle et $\mathbf{J}$. Vouidibio pour avoir capturé les drosophiles; J. Estève, J.P. CROzE et M. PAGÈs pour leurs remarques concernant le traitement statistique des données, et M. Boulétreau et C. Bí́mont pour leurs critiques du manuscrit.

\section{Références bibliographiques}

Allemand R., 1976 a. Importance adaptative du rythme circadien de ponte chez les drosophilidés : comparaison de huit espèces du genre Zaprionus. C.R. Acad. Sci. Paris, D 282, 85-88.

Allemand R., 1976 b. Les rythmes de vitellogenèse et d'ovulation en photopériode LD 12:12 de Drosophila melanogaster. J. Insect Physiol., 22, 1031-1035.

Allemand R., 1977. Influence de l'intensité d'éclairement sur l'expression du rythme journalier d'oviposition de Drosophila melanogaster en conditions LD 12:12. C.R. Acad. Sci. Paris, D 284, 1553-1557. 
Allemand R., 1983 a. The circadian oviposition rhythm of Drosophila melanogaster : influence of the laying substrate and of experimental conditions. Biol. Behav., 8, 231-245.

Allemand R., $1983 \mathrm{~b}$. The circadian oviposition rhythm of Drosophila melanogaster : influence of biotic factors. Biol. Behav., 8, 273-288.

Allemand R., David J., 1976. The circadian rhythm of oviposition in Drosophila melanogaster : a genetic latitudinal cline in wild populations. Experientia, 32, 1403-1404.

Allemand R., David J.R., 1984. Genetic analysis of the circadian oviposition rhythm in Drosophila melanogaster : effects of drift in laboratory strains. Behav. Genet., sous presse.

BENZECRI J.P., 1973. L'analyse des données. II. - L'analyse des correspondances. 619 p., Dunod, Paris.

Boulétreau-Merle J., Allemand R., Cohet Y., David J.R., 1982. Reproductive strategy in Drosophila melanogaster : significance of a genetic divergence between temperate and tropical populations. Oecologia, 53, 323-329.

Bunning E., 1973. The physiological clock. 3rd ed., 258 p., English University Press, London.

Chessel D., Lebreton J.D., Prodon R., 1982. Mesures symétriques d'amplitude d'habitat et de diversité intra-échantillon dans un tableau espèces-relevés : cas d'un gradient simple. C.R. Acad. Sci. Paris, III 295, 83-88.

Clayton D.L., Paietta J.V., 1972. Selection for circadian eclosion time in Drosophila melanogaster. Science, 178, 994-995.

Dagnelie P., 1975. Analyse statistique à plusieurs variables. 362 p., Presses Agronomiques de Gembloux.

David J., 1970. Le nombre d'ovarioles chez Drosophila melanogaster : relation avec la fécondité et la valeur adaptative. Arch. Zool. Exp. Gen., 111, 475-489.

DAVID J.R., 1979. Utilization of morphological traits for the analysis of genetic variability in wild populations. Aquilo Ser. Zool., 20, 49-61.

David J., Foulllet P., 1973. Enregistrement continu de la ponte chez Drosophila melanogaster et importance des conditions expérimentales pour l'étude du rythme circadien d'oviposition. Rev. Comp. Anim., 7, 197-202.

De Prins J., Cornelissen G., Hillman D., Halberg F., van Dijck C., 1981. Harmonic interpolation yields paraphases and orthophases for biologic rhythms. Proceedings of the XIIIth International Conference of the International Society for Chronobiology, Pavia, September 4-7, 1977, Halberg F., Schewing L.E., P. Well E.W., Hayes D.K. (ed.), 333-344.

Fontdevilla A., Ruiz A., Ocana J., Alonso G., 1982. Evolutionary history of Drosophila buzzatii. II. - How much has chromosomal polymorphism changed in colonization ? Evolution, 36, 843-851.

Gauch H.G. Jr., 1982. Multivariate analysis in community ecology. 298 p., Cambridge University Press, Cambridge.

Gloagen J.C., Gautier N., 1981. Pattern development of the vegetation during colonization of a burnt heathland in Brittany (France). Vegetatio, 46, 167-176.

Grantham R., Gautier C., Gouy M., Jacobzone M., Mercier R., 1981. Codon catalog usage in a genome strategy modulated for gene expressivity. Nucleic Acids Research, 9, 43-74.

Hardeland R., Stange G., 1973. Comparative studies of the circadian rhythm of locomotor activity of 40 Drosophila species. J. Interdiscipl. Cycle Res., 4, 353-359.

Hugelin A. (ed.), 1982. Comptes rendus du Colloque National : "Mécanismes des rythmes biologiques : concepts, structures, modèles». Seillac, 28 septembre - 2 octobre 1982, $155 \mathrm{p}$.

KaWANishi M., Watanabe T.K., 1978. Difference in photo-preferences as a cause of coexistence of Drosophila simulans and D. melanogaster in nature. Jap. J. Genet., 53, 209-214.

KoNopKA R.J., 1979. Genetic dissection of the Drosophila circadian system. Federation Proc., 38, 2602-2605. 
KonOPKA R.J., BENZER S., 1971. Clock mutants of Drosophila melanogaster. Proc. Natl. Acad. Sci. USA, 68, 2112-2116.

LEgendre L., Legendre P., 1979. Ecologie numérique. Tome 2 : La structure des données écologiques. 247 p., Masson et PUF, Paris.

Martin E., 1982. Distribution des activités comportementales chez le triton alpestre Triturus alpestris en phase aquatique : étude de la variabilité interindividuelle et de l'intensification locale. Thèse Doctorat $3^{\circ}$ cycle, Lyon, $237 \mathrm{p}$.

PARsons P.A., 1980. Isofemales strains and evolutionary strategies in natural populations. In : Hecht et al. (ed.), Evol. Biol., 13, 175-217.

PItTendrigh C.S., 1967. Circadian systems I : the driving oscillation and its assay in Drosophila pseudoobscura. Proc. Natl. Acad. Sci. USA, 58, 1762-1767.

Rivault C., 1981. Rôle des facteurs sociaux sur l'expression de la rythmicité circadienne dans un groupe chez Periplaneta americana (Dictyoptère). Behaviour, 77, 23-43.

Saunders D., 1982. Insect clocks. 2nd ed., 409 p., Pergamon Press, Oxford. 